\title{
Creation of high-performing medical department
}

\author{
David Thomas Neilipovitz, John Kim \\ The Ottawa Hospital, University of Ottawa, Canada
}

Received: October 8, 2021

DOI: $10.5430 /$ jha.v10n5p18
Accepted: October 24, $2021 \quad$ Online Published: November 1, 2021

URL: https://doi.org/10.5430/jha.v10n5p18

\begin{abstract}
Background: Transforming dysfunctional medical groups into high-performing departments is a process that physician leaders are not typically trained to enact. Multiple issues challenge the ability to successfully create a financially sound department that offers high-quality care along with impactful academic deliverables.

Methods: We present an example of a critical care group that was highly dysfunctional that was transformed into a highperforming medical department. It underwent a change that was achieved through three stages: (1) Defining Purpose; (2) Relationship Building and Problem Solving; and (3) Group Development. The later stage is approached in a three-phase cycle. Results: Success was achieved on all deliverables including clinical care, academics and finances as validated by external measures. The department was awarded best practice for delivery of clinical care by an international accreditation group. It was twice recognized as their hospital's highest engaged medical group. Academic deliverables increased to become a high performer all while financial stability was achieved. The importance of health and wellness is highlighted.

Conclusions: The process for transforming departments is suggested in a step-wise approach for other groups to achieving similar success.
\end{abstract}

Key Words: Medical departments, High-performing, Academics, Transforming

\section{INTRODUCTION}

Expectations of a modern health care system is that medical groups operate with high efficiency but are highly effective and adaptable to unforeseen problems including the COVID pandemic. Typical physician training lacks fundamental administrative skills to achieve these goals. Although leadership programs are available for physician, we were unable to find specific strategies for managing a medical department/division. Compounding to this paucity of information is the nuance of the challenges of the Canadian health care system which is a decentralized system of health financing and delivery unlike most other countries. ${ }^{[1]}$

Since there is not a uniformly accepted definition of a highperforming medical group, ${ }^{[2]}$ we choose the Commonwealth
Fund Commission definition which is a group that offers high-quality, safe care; access for all people; efficient, highvalue care; with the capacity to improve. ${ }^{[3]}$ We present how we transformed our group from a dysfunctional group into an engaged, cohesive, high-performing department.

\section{METHODS}

Our group experienced problems from its inception in 1999 when our Department of Critical Care was created by amalgamating two culturally distinct academic intensive care units (ICUs) at separate hospitals. Initially 10 ICU specialists (called intensivists) were split equally between facilities with no one practicing at both campuses despite similar university appointments. Each campus operated independently with different clinical and business models with distinct cul-

*Correspondence: David Thomas Neilipovitz; Email: dneilipovitz@toh.ca; Address: The Ottawa Hospital, University of Ottawa, Canada. 
tural differences. Remuneration for all was predominately a fee-for-service reimbursement from the provincial insurance system. As is typical in Canada, the department functions as a group of peers with limited hierarchal structure.

The inaugural Head was denied reappointment due to negative feedback from the campus where they did not practice. Several interim heads filled the leadership void. Despite shared recruitment for new appointees, continued conflict between sites on clinical, business and operational models persisted. In 2005, an external Department Head was recruited. Several retreats were held to create a vision and a shared business model. Unfortunately, divisions persisted along with overdrawn budgets which contributed to the Head leaving their position after three years.

After an extended search, in 2011 a relatively junior member was selected as the new Head. At this time, the department had 17 intensivists with approximately $30 \%$ practicing at a single site and $40 \%$ working at both. The Head's first initiative was to start the process of group unification. The following stages were identified: (1) Define Purposes; (2) Problem Solving; and (3) Group (Re)Development.

\subsection{Stage 1 - Defining purpose}

Before departmental transformation, we asked what is the group's purpose? Simply put, the primary purpose is to care for patients. ${ }^{[3]}$ Our group unanimously agreed to state our primary responsibility was to deliver clinical care of the highest possible standard that was evidence-based, ethically-sound, compassionate and reliable (see Table 1). After this, three secondary purposes were identified- research, education and financial sustainability with an eventual fourth being member health and wellness.

Table 1. Proposed purposes of a medical group

\begin{tabular}{ll}
\hline & Items \\
\hline Primary & High Quality Medical Care \\
Secondary & Health and Wellness of Group Members \\
& Financial Prosperity \\
& Education Mission \\
& Research Mandate \\
\hline
\end{tabular}

2.2 Stage 2 - Relationship building and problem solving After defining purposes, the focus was to create constructive relationships between group members. The absence of such relationships was limiting group cohesion and its ability to develop. Impediments to group cohesion were identified by group consensus. Issues such as a lack of formal voting processes or dispute resolution mechanisms were limiting departmental activity. Our group benefited from insights from two external reviews but we still went through the vitally

Published by Sciedu Press important internal step of self-identifying all concerns as inevitably some problems are not shared in external reviews. Identified problems were primarily: (1) Poor communication; (2) Mistrust between campuses; and (3) Perceived lack of collegial respect.

As communication is recognized as central to conflict resolution, ${ }^{[4,5]}$ our focus became to create relationships through open and transparent communication. Relationship building was facilitated with the department head meeting with each member individually and in small groups. Open office time with the head was shared with all. The emphasis was to increase trust in the group's direction along with creation of an inclusive atmosphere. Further steps included increasing transparency and greater inclusion in decisionmaking. Demonstration of these principles were reflected in the understanding that no major decisions were made without universal communication and presentation of issues without solutions to the group. Perhaps inefficient and time consuming, these approaches were important to create trust and establish mutually respectful relationships.

Surprisingly, the department never had annual reappointment interviews which was rectified. Increased frequency of departmental meetings of shorter duration with structure conforming to recommended practices ${ }^{[6]}$ was established. Limiting email to information distribution without using it as a substitute for group meetings to make decisions was helpful. We established an Annual General Meeting (AGM) with an Annual Report. The AGM serves to review the prior year's goals, assess present state and to celebrate achievements. Failure to celebrate success or review progress we believe introduces discontent and a sense of futility. Our final important relationship building process to improve group cohesion was to foster the development of a new shared culture. An effective approach for us was to emphasize participation in our social gatherings including family-based activities.

\subsection{Stage 3 - Group (re)development}

Once direction on group transformation was set, the path to this was divided into stages or phases (see Table 2). Each phase with projected timelines for implementation were presented. A key element was to frequently present a "current snapshot" of where the department was in terms of each phase including an annual evaluation of progress for each phase of (re)development. This approach helps engage members who were skeptical or even may not have been fully supportive of an initiative. Our group had members who were skeptical of combining group research budgets because of perceived differences. Thus, demonstrating that production increased at both campuses not only confirmed the decision but provided impetus to change and address 
other issues. Repeated success and transparency in the group (re)development phase allowed us to overcome inherent cynicism.

Table 2. Phases of a group development

\begin{tabular}{l}
\hline Phases \\
\hline Solidify Foundation \\
Set Direction \\
Actions-Celebrations-Reflections \\
\hline
\end{tabular}

An important albeit obvious caveat we offer is to not be overly ambitious in goals and to set realistic timelines. It is perhaps fashionable that new leaders set "first 100 days"[7] goals and other in-vogue rapid interventions; we believe that in many cases, this is an unrealistic and potentially damaging strategy. ${ }^{[8,9]}$ Perhaps for groups in crisis a shorter timeline with defined goals is warranted, we suspect however it is not often that such drastic approaches are helpful. In our case, with over 10 years of failed interventions, false starts and no perceived immediate clinical crises, rapid changes were not necessary and could have been detrimental. We believe sustained change takes time to achieve. Any rapid interventions for immediate success are likely to be transient in nature, with group members likely to revert to previous patterns of behaviour. Furthermore, the consequences of such failures would be disengaging and potentially encourage individuals to again choose their own approaches. We therefore choose to undertake the following phases for our group's (re)development:

\section{Phase I - Solidifying Foundation}

The first phase we recommend is ensuring there is a solid "foundation". A department requires a supporting infrastructure including adequate support staff, office space and equipment. It is essential for there to be a framework that the group abides too. Our group worked to create its agreements that clearly addressed how the group would interact and established practices and defined responsibilities (e.g. academia). Our group's association agreement is the most important as it details meeting rules, voting processes, conduct, privileges and other expectations. The final aspect of solidifying a group's foundation is a strategy to ensure financial stability. A practice plan detailing financial oversight with annual budgets from a representative finance committee that has transparent oversight was essential. Our group decided that the Head would have an arms-length relationship with the finance committee as a non-voting member.

Physicians may find financial issues unsavoury but sound financial practice is essential for departmental success. To solidify our financial foundation, we first agreed to the principles that long-term stability and group equitability were our shared goals. We also agreed that yearly deficits requiring end-of-year payments could not continue thus requiring our group to budget responsibly while focusing on securing departmental funds.

Financial stability was obtained by agreeing to fixed yearly tithe rate of $5 \%$ on all clinical earnings. Academic centres such as ours receive some government funding through an academic funding plan which has a value roughly a tenth of the fee-for-service amount. The department agreed that academic funding would go to the departmental account rather than be distributed directly to members. Together these funds significantly increased the yearly departmental income. For us equitability meant each physician would receive similar compensation for similar work performed. Our physicians would receive a similar income for each day worked and comparable on-call income. Importantly, call requirements were shared equally by all members regardless of experience. As physician remuneration in Ontario is predominately fee-forservice, our balancing required sharing of billing. Monthly and annual billing statements for all members were published to the group, to reinforce the commitment to transparency and equitability. Bad debts were shared by all members and incorporated into the balancing.

\section{Phase II - Set direction}

Once foundation was addressed the group sets 2-year priorities for its clinical and its four non-clinical missions. Clinically we focused on three foci (ultrasound, patient mobilization, improved care transitions) that we as group were committed to addressing. For academics we set targets for grants, study enrollment and publications that were challenging but feasible. To help achieve our group's primary purpose, we created a Quality and Safety Group (QuSa) which was novel at the time. Our commitment to QuSa included recruiting staff who had training to both measure and improve safety and clinical performance. For finances we focused on transparency but strived for stability. We applied a uniform tithe on clinical earnings and agreed that all nonclinical income went to the group. One of our early failings that we subsequently addressed was to add a fourth non-clinical priority which was to address members' Health and Well-Being.

As income in our model was dependent on number of weeks worked, the group agreed to a set distribution of weeks to physicians. Historical allocations of senior members having more weeks than junior members was identified as an issue requiring addressing. We set direction to have these discrepancies disappear over five years to allow for smoothing, natural attrition through retirement and adaptation by senior physicians finding alternative income sources. 


\section{Phase III - Actions-celebrations-reflections}

Phase III is the stage to make things happen. Streamline committees and distributing responsibilities to leads for each pillar (Clinical, Education, Research, QuSa, Health) was established. At each monthly meetings the leads would keep the group current with each pillar. This shares ongoing challenges but also provides opportunity to celebrate successes. Complementing these updates is the annual report documenting achievements which culminates with the AGM. Annual reports allow referencing of achievements and can be distributed for departmental promotion.

Each AGM requires leads to present on yearly achievements while comparing results to the targets set the year before. Discussions on each pillar culminates in the setting targets for the upcoming year. These annual reviews enhance accountability but also provides some flexibility to adjust and revise future goals based on new or changing priorities in each pillar. The AGM also ensures a group celebrates its successes but also refines group's plan for development. A final element in the third phase involves succession planning.

\section{Results}

In 3-years our approach produced significant results. Clinically our department was recognized as a top-10th percentile performing group by Accreditation Canada. It was consistently the highest rated clinical rotation for residents. Academic production as measured by peer-reviewed publications more than doubled yearly (see Figure 1) while grants increased in spite losing an internationally renowned researcher and an austere financial environment (see Figure 2). Financially we had positive balances even though expenditures for academia and other non-clinical activities (e.g. leadership development, physician education, staff wellness) increased expenses by more than $100 \%$. The contentious issue of finances disappeared as reflected by unanimous approval of annual budgets and creation of healthy reserves.

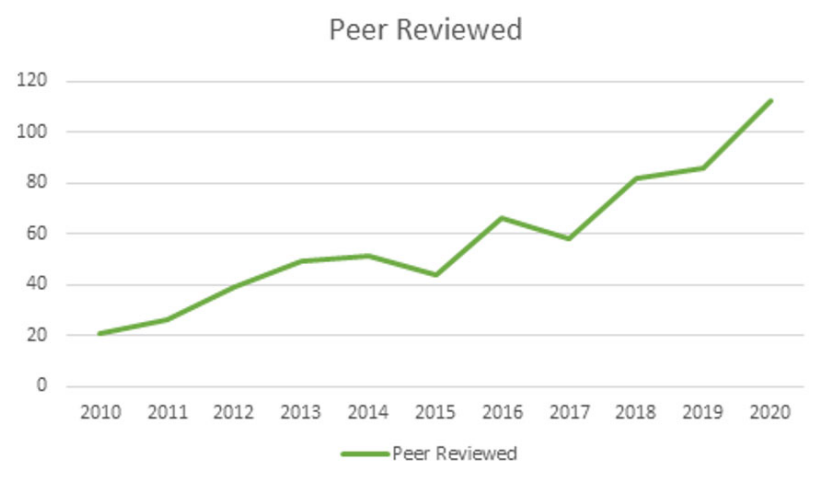

Figure 1. Flow chart of the yearly number of peer reviewed publications

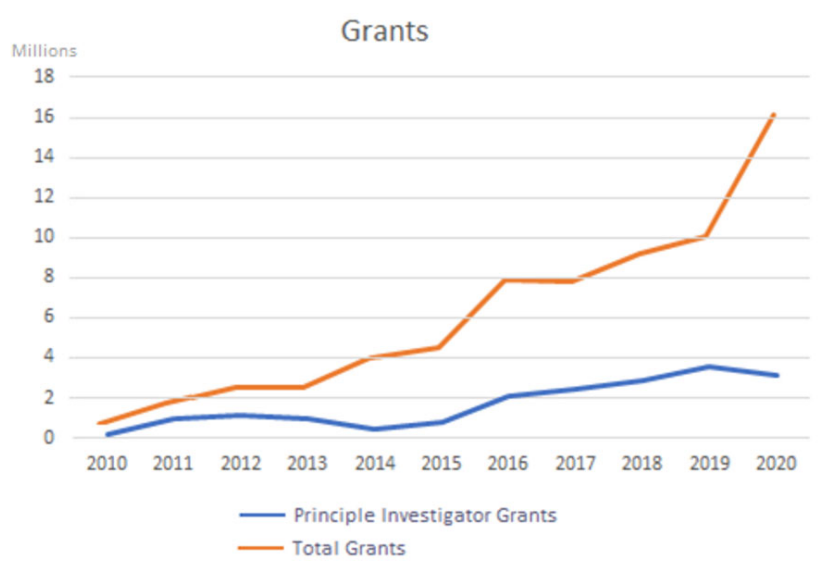

Figure 2. Flow chart of the yearly values of research grants for principle investigator and department total

The longevity of these changes is demonstrated by sustained growth of all pillars for the subsequent 8-year period. The Department received a best practice recognition for clinical care with family communication from Accreditation Canada. Despite the group size remaining consistent the academic production continued to increase annually with over 100 peerreviewed publications and grants of over 3.5 million annually becoming one of the university's highest performer when adjusted for department size. The financial reserves were used to create new Academic Chair positions and initiatives.

The healthiness of a group however is not simply reflective on its clinical deliverables and productive capacity. Physician engagement is an important factor for improving performance.$^{[9-11]}$ Our department had the highest rate of engagement when our institution performed engagement surveys using Aon Hewitt Survey. In 2019 the Ontario Hospital Association performed a survey of 39 Ontario hospitals to generate a composite engagement score from 3 components including vigor, dedication and absorption. Our Critical Care group was above provincial means and our composite value of 4.57 out of 7 was well above the provincial average of $4.15 .^{[12]}$

\section{Discussion}

This case reflects a successful change management in transforming a dysfunctional and divided medical group into a high-performing department. Concepts key to successful organizational development are documented in health care literature. ${ }^{[9-11,13-16]}$ Some of these concepts are challenging to enact in a system that operates as a flattened hierarchy. Our example however confirms many existing change management and engagement strategies ${ }^{[15,16]}$ are adaptable to still be effective in our non-hierarchal Canadian centre.

We believe some contributors for success was a leadership 
team made up of representation from all sites but all demographics as well. Establishing a new culture rather than adoption of one over another is essential. Culture is a complex entity particular in the context of healthcare. ${ }^{[17]}$ Even in one city, the culture can vary from one centre to another as it reflects difference in language, heritage, training, religion and other factors. ${ }^{[17]}$ Our strategy was to not focus on differences but to establish common goals, values and process. We insisted on supporting holiday get-togethers with group members and their families along with other social events. Although these could be considered as frivolous unnecessary expenditures, the value is to demonstrates commonality and promote relationship building. Thus, with transparent communication and relationship building we had complete transformation of our dysfunctional team into a high-performing group. Contrast to our success was the years previous which lacked both a focus on relationships and on communication.

The values of selecting an internal versus external heads in change management is debated. ${ }^{[18]}$ Internal heads have ideally been groomed and benefit from having existing relationships and a greater understanding of the issues affecting a group. The selection of an external candidate is often performed when teams are under performing or in difficult situations even though the success of such a strategy is questionable and not reliably efficacious. ${ }^{[18]}$ While an external Head offers the ability to offer an outside perspective and challenge the status quo, they also lack an understanding of the organizational history and personnel. ${ }^{[19]}$ In our case, the internal Head chosen to succeed lacked experience, but had the built-in advantages of homophily (similar clinical profile and background), linkage (already collaborating with many members) and provided energy and synergy in rallying the group to agreed-upon priorities and goals. The familiarity internal Heads have with organizational operations also enable a structuring advantage in being able to effect change at a Hospital level. ${ }^{[19]}$ We believe our example demonstrates that solutions are often available internally and more reflective of processes than individuals.

The processes we present may appear to be self-evident but had not been previously adopted. We suspect that many underperforming medical departments suffer from problems with trust, communication and lack of constructive relationships. As it takes times to address these problems it is prob- lematic to emphasis the approach of "quick wins" and the first 100 days. ${ }^{[8-10]}$ Had our group with its history of multiple failed attempts at change management solely focused on short-term wins we would likely have failed again. Nonetheless we included some quick wins as they are important as they create some positive atmosphere thus encouraging groups to pursue allow longer-term but more formidable changes. ${ }^{[20]}$ Equally if not more important, we celebrated the quick wins to emphasize the potential for long-term change. It is important to recognize obstacles and setbacks will and did occur. The sudden death of a colleague highlighted our group's inattention to health and well-being as a pillar of group development. Our painful lesson is best avoided as staff wellness is now well recognized as crucial for creation of a high functioning department. ${ }^{[21]}$ The other pillar that is vital but may be deemed unsavoury is financial success. Recognizing both the importance of financial success and ensuring its achievement is fundamental to a group's transformation. Change will not happen if members are worrying or experiencing financial uncertainty or distress.

There are important limitations to this case and the advocated approaches. Our approaches were selected given our centralized health care system where organizational performance is not directly linked to individual employment or remuneration. In our health care system, a flatter hierarchy results in teams operating more as a group of peers thus making consensus building essential to promote group change. Whether such an approach is directly applicable in systems with a more defined hierarchy we can only speculate if the approach would be as effective. Although our approach may not be completely effective for groups in acute crisis or collapse given the time required to obtain results, it would be required in part to ensure stability and future growth.

\section{Conclusions}

Transforming a Canadian health care medical group into a high-performing group presents a unique challenge given the unique characteristics present in a decentralized health care system that operates within a flattened hierarchy. We present this case to outline an operational plan for successful change management in such a system.

\section{CONFlicts OF INTEREST Disclosure}

The authors declare they have no conflicts of interest. 


\section{REFERENCES}

[1] Dickson G. Anchoring Physician Engagement in Vision and Values: Principles and Framework. Regina: Regina Qu'Appelle Health Region; 2012. Available from: chrome-extension://efaidnbm nnnibpcajpcglclefindmkaj/viewer.html?pdfurl=https $\%$ $3 \mathrm{~A} \% 2 \mathrm{~F} \% 2 \mathrm{Fww}$.rqhealth. ca $\% 2 \mathrm{Fservice-lines} \% 2 \mathrm{Fmaster} \%$ 2 Ffiles $\% 2$ Fanchoring . pdf\&clen=1276119\&chunk=true

[2] Aluwhalia SC, Damberg CL, Silverman M, et al. What Defines A High-Performing Health Care Delivery System: A Systematic Review. The Joint Commission Journal on Quality and Patient Safety. 2017; 43: 450-459. PMid: 28844231. https://doi.org/10.101 6/j.jcjq.2017.03.010

[3] Shih A, Davis K, Schoenbaum S. Organizing the US Health Care Delivery System for High Performance. New York, NY: Commonwealth Fund; 2008. Available from: https://www.commonwealthfund .org/publications/fund-reports/2008/aug/organizing -us-health-care-delivery-system-high-performance

[4] Marshall P, Robson R. Conflict Resolution. Royal College of Physicians and Surgeons of Canada Bioethics Primer. Available from: http://www.royalcollege.ca/rcsite/bioethics/p rimers/conflict-resolution-e

[5] Mayer B. The dynamics of conflict resolution: A practitioner's guide to clear thinking. San Francisco: Jossey-Bass; 2000. Available from: chrome-extension://efaidnbmnnnibpcajpcglclefindmk aj/viewer. html?pdfurl=http $\% 3 \mathrm{~A} \% 2 \mathrm{~F} \% 2 \mathrm{Forgwise}$. ca $\% 2 \mathrm{Fsi}$ tes $\% 2$ Fosi.ocasi.org.stage $\% 2$ Ffiles $\% 2$ Fresources $\% 2$ FTh e\%2520Dynamics\%2520of\%2520Conflict\%2520Resolution. pdf\&clen=36214\&chunk=true

[6] Robert HM. Robert's Rules of Order Revised. 1915 (Public Domain). Available from: http://www.rulesonline.com/rror--00.ht $\mathrm{m}$

[7] Watkins MD. Why the First 100 Days Matters. Harvard Business Review. 2009. Available from: https://hbr.org/2009/03/wh y-the-first-100-days-matters

[8] Torres R, Tollman P. Five Myths of a CEO's First 100 Days. Harvard Business Review. 2014. Available from: https ://hbr.org/2012 /01/five-myths-of-a-ceos-first-100

[9] Kotter JP. Leading Change. Boston: Harvard Business School Press; 1996.

[10] Phelan D. Best Practice Change Management Guidelines. National Health Service. 2010. Available from: https://pdf4pro.com/view/best-practice-change-m anagement-guidelines-v1-4eb50c.html
[11] Lukas CV, Holmes SK, Cohen AB, et al. Transformational change in health care systems: an organizational model. Health Care Management Review. 2007; 32(4): 309-320. PMid: 18075440. https : //doi.org/10.1097/01.HMR.0000296785.29718.5d

[12] Perreira T, Prokopy M, Brown A, et al. Measuring physician engagement in quality improvement: a pilot study. Can J Physician Leadership. 2020; 6(3).

[13] Isles V, Sutherland K. Organizational change: A review for health care managers, professionals and researchers. London: National Co-ordinating Centre for NHS Service Delivery and Organization. Available from: https://pdf4pro.com/view/organisationa 1-change-netscc-ac-uk-29718.html

[14] Lewin K. Field Theory in Social Science: Selected Theoretical Papers. Kurt Lewin Ed. Dorwin Cartwright, Boston, Massachusetts: MIT Research Center for Group Dynamics and New York: Harper and Brothers Publishers; 1945.

[15] Langley GL, Moen R, Nolan KM, et al. The Improvement Guide: A Practical Approach to Enhancing Organizational Performance (2nd edition). San Francisco: Jossey-Bass Publishers; 2009.

[16] Mannion R, Davies H. Understanding organisational culture for healthcare quality improvement. BMJ. 2018; 363: k4907. PMid: 30487286. https : //doi .org/10.1136/bmj .k4907

[17] Dickson G, Lindstrom R, Black C, et al. Evidence-informed management in Canadian Healthcare organizations. Canadian Health Services Research Foundation. 2012. Available from: https : //ww w.deslibris.ca/ID/234417

[18] Harrell E. Succession Planning: What the Research Says Harvard Business Review. 2016; 12: 70-74. Available from: https://hbr.org/2016/12/succession-planning-what-t he-research-says

[19] Lunenberg FC. Managing Change: The Role of the Change Agent Int J Manag Bus Adm. 2010; 13(1): 1-6.

[20] Appelbaum SH, Habashy S, Malo J, et al. Back to the future: revisiting Kotter's 1996 change model. J of Management Development. 2012; 31(8): 764-782. https://doi.org/10.1108/0262171121 1253231

[21] Tu HT, Mayrell RC. Employer Wellness Initiatives Grow Rapidly, but Effectiveness Varies Widely. NIHCR Research Brief No. 1, July 2010. Available from: https://www.nihcr.org/analysis/imp roving-care-delivery/prevention-improving-health/ employer-wellness-initiatives/ 